\title{
A PROPERTY OF PROJECTIVE IDEALS IN SEMIGROUP ALGEBRAS
}

\author{
WILLIAM R. NICO ${ }^{1}$
}

\begin{abstract}
The condition that certain left ideals in a finite monoid generate projective left ideals in the semigroup algebra imposes a strong restriction on the intersection of principal left ideals in the semigroup.
\end{abstract}

Let $S$ be a finite monoid, $k$ be a commutative ring with identity, and let $I \subset S$ be a left ideal in $S$. We demand that $k I$ be projective as a left $k S$ module and investigate the resulting restrictions on the structure of $I$. In particular we can look for necessary conditions on $S$ for $k S$ to be left hereditary. (A sufficient condition is obtained in [4] and [5].) Semigroup terminology below follows [1] and [2].

We first need the following facts, which are valid in any ring with identity.

LEMMA 1. Let $R$ be a ring with identity. Let $I \subset R$ be a left ideal which is projective as a left $R$-module. Let $e \in R$ be any idempotent. Then the left ideal $I+R e$ is projective if and only if $I \cap R e$ is a direct summand of $I$.

Proof. We observe that we have the following two short exact sequences:

$$
\begin{aligned}
& 0 \rightarrow I(1-e) \rightarrow I+R e \rightarrow R e \rightarrow 0, \\
& 0 \rightarrow I \cap R e \rightarrow I \rightarrow I(1-e) \rightarrow 0,
\end{aligned}
$$

where the map on the right end of the first sequence is $x \mapsto x e$, which has kernel $(I+R e) \cap R(1-e)=I(1-e)$, and the map on the right end of the second sequence is $x \mapsto x(1-e)$. Since $R e$ is projective, the first sequence always splits, so that $I+R e$ is projective if and only if $I(1-e)$ is. On the other hand, since $I$ is projective, $I(1-e)$ is projective if and only if the second sequence splits.

Received by the editors June 5,1972 .

AMS (MOS) subject classifications (1970). Primary 20M10, 20M25; Secondary 16A32, 16A50, 16A60.

Key words and phrases. Semigroup, monoid, semigroup algebra, projective modules, idempotents, left ideals.

1 This research supported in part by the National Science Foundation.

(C) American Mathematical Society 1973 
COROLlARY. If $R$ is a ring with identity and if $e_{1}$ and $e_{2}$ are idempotents of $R$, then $R e_{1}+R e_{2}$ is a projective left ideal of $R$ if and only if $R e_{1} \cap R e_{2}$ has an idempotent generator.

Proof. By the lemma, $R e_{1} \cap R e_{2}$ is a direct summand of $R e_{1}$. If $\pi$ : $R e_{1} \rightarrow R e_{1} \cap R e_{2}$ is a retraction of $R e_{1}$ onto $R e_{1} \cap R e_{2}$, then $f=\pi\left(e_{1}\right)$ is the desired idempotent since $f^{2}=f \pi\left(e_{1}\right)=\pi\left(f e_{1}\right)=\pi(f)=f$ and $R f=\pi\left(R e_{1}\right)=$ $R e_{1} \cap R e_{2}$.

Now let $S$ be a finite monoid, $e_{1}, e_{2} \in S$ be idempotents, and $k$ be a commutative ring with identity. Then we have the following:

THEOREM 1. (i) If the left ideal $k S e_{1}+k S e_{2} \subset k S$ is projective as a left $k S$-module, then the semigroup left ideal $S_{1} \cap S e_{2} \subset S$ is generated by idempotents of $S$, or $\mathrm{Se}_{1} \cap \mathrm{Se}_{2}=\varnothing$.

(ii) Furthermore, if $L$ is an $\mathscr{L}$-class of $S$ maximal in the $\mathscr{L}$-class ordering with respect to the property that $L \subset S_{1} \cap S_{2}$ and if $J$ is the $\mathscr{J}$-class (= $\mathscr{D}$-class) of $S$ containing $L$, then

(a) $J$ is a regular $\mathscr{J}$-class of $S$, and

(b) if $J^{0} \approx \mathscr{M}^{0}(G ; I, \Lambda, P)$ is a Rees matrix representation of $J^{0}$ with maximal subgroup $G$, index sets I and $\Lambda$ (for the $\mathscr{R}$ - and $\mathscr{L}$-classes, respectively), and sandwich matrix $P$, if $\Lambda^{\prime}$ is the subset of $\Lambda$ which corresponds to the set of $\mathscr{L}$-classes of $J$ which satisfy the above maximality condition, and if $\mathscr{M}^{0}\left(G ; I, \Lambda^{\prime}, P^{\prime}\right)$ is the correspondingly restricted Rees matrix semigroup, then $P^{\prime}$ has a right inverse as a matrix over $k G$.

Proof. By the corollary to Lemma 1 , there is some $\gamma=\gamma^{2} \in k S e_{1} \cap$ $k S e_{2}$ such that $k S e_{1} \cap k S e_{2}=k S \gamma$. We observe also that $k S e_{1} \cap k S e_{2}=$ $k\left(S e_{1} \cap S e_{2}\right)$. Suppose $S e_{1} \cap S e_{2} \neq \varnothing$, and let $L$ be an $\mathscr{L}$-class of $S$ maximal with respect to $L \subset S e_{1} \cap S e_{2}$. Let $U=\{x \in S: S x \not L\}$. Then $U$ and $U \cup L$ are left ideals of $S, U \cap L=\varnothing$, and the maximality of $L$ implies that $S e_{1} \cap S e_{2} \subset U \cup L$.

Now one can write $\gamma=\gamma_{L}+\gamma_{U}$ where $\gamma_{L}$ is a $k$-linear combination of elements of $L$ and $\gamma_{U}$ is a $k$-linear combination of elements of $U$. If $s \in$ $L \subset S e_{1} \cap S e_{2}$, then $s=s \gamma=s \gamma_{L}+s \gamma_{U}$, where $s \gamma_{L}$ is a $k$-linear combination of elements of $L^{2}$ and $s \gamma_{U}$ is a $k$-linear combination of elements of $U$. Since the elements of $S$ are linearly independent over $k$, this implies that $L \subset L^{2}$. If $J$ is the $\mathscr{J}$-class containing $L$, then $J \cap J^{2} \neq \varnothing$. Hence $J$ is regular, and thus $L$ contains an idempotent. Since $S e_{1} \cap S e_{2}=\bigcup\{S L: L \subset S$ is a maximal $\mathscr{L}$-class as above\}, we have that $S e_{1} \cap S e_{2}$ is generated by idempotents.

Let $J^{0} \approx \mathscr{M}^{0}(G ; I, \Lambda, P)$; let $\left\{L_{\lambda}: \lambda \in \Lambda\right\}$ be the $\mathscr{L}$-classes of $J$; and let $\left\{L_{\lambda}: \lambda \in \Lambda^{\prime}\right\}, \Lambda^{\prime} \subset \Lambda$ be the set of $\mathscr{L}$-classes of $J$ maximal in the $\mathscr{L}$-class ordering with respect to $L_{\lambda} \subset S e_{1} \cap S e_{2}$. Then we let $P^{\prime}$ be the corresponding 
$\left|\Lambda^{\prime}\right| \times|I|$ submatrix of the $|\Lambda| \times|I|$ matrix $P$ and see that the Rees matrix semigroup $\mathscr{M}^{0}\left(G ; I, \Lambda^{\prime}, P^{\prime}\right)$ is isomorphic to $V^{0}$ where $V=\bigcup\left\{L_{\lambda}: \lambda \in \Lambda^{\prime}\right\}$.

Let $W=\{x \in S: S x \cap V=\varnothing\}$. Then $W$ and $W \cup V$ are left ideals of $S$, $W \cap V=\varnothing$, and $S e_{1} \cap S e_{2} \subset W \cup V$. We can write $\gamma=\gamma_{V}+\gamma_{W}$ with $\gamma_{V}$ (respectively $\gamma_{W}$ ) a $k$-linear combination of elements of $V$ (respectively $W$ ). If we write $\gamma_{V}$ as an $|I| \times\left|\Lambda^{\prime}\right|$ matrix $Q$ over $k G$, then the fact that $s \gamma_{V}=$ $s \gamma-s \gamma_{W}=s-s \gamma_{W}$ for all $s \in V$, and $s \gamma_{W} \in k W$, implies that the matrix product $P^{\prime} Q$ is a $\left|\Lambda^{\prime}\right| \times\left|\Lambda^{\prime}\right|$ identity matrix.

COROLlaRY 1. In the situation of (ii) above, it must be that $|I| \geqq\left|\Lambda^{\prime}\right|$.

Proof. If we extend the augmentation homomorphism $k G \rightarrow k$, given by $g \mapsto 1$ for all $g \in G$, to matrices over $k G$, then since $P^{\prime} Q$ is the identity, the rank of the image of $P^{\prime}$ must be $\left|\Lambda^{\prime}\right|$, which implies that $|I| \geqq\left|\Lambda^{\prime}\right|$.

COROLlARY 2. No two rows of $P^{\prime}$ have zero and nonzero entries in exactly the same locations.

Proof. If $P^{\prime}$ had two such rows, then the rank of the image of $P^{\prime}$ under the augmentation homomorphism would be less than $\left|\Lambda^{\prime}\right|$, impossible.

COROLlaRY 3. If $S$ is a union of groups semigroup, if $e_{1}$ and $e_{2} \in S$ are idempotents, and if $k S e_{1}+k S e_{2}$ is $k S$-projective, then if $S e_{1} \cap S e_{2} \neq \varnothing$, the $\mathscr{L}$-classes $L$ of $S$ maximal with respect to $L \subset S e_{1} \cap S e_{2}$ all lie in distinct $\mathscr{J}$-classes.

Proof. If $S$ is a union of groups then all the entries of $P$ and $P^{\prime}$ above must be nonzero. Thus by Corollary $2, P^{\prime}$ has only one row, that is, $\left|\Lambda^{\prime}\right|=1$, which says that only one $\mathscr{L}$-class $L$ of $J$ is maximal with respect to $L \subset$ $S e_{1} \cap S e_{2}$.

THEOREM 2. Let $S$ be a union of groups monoid such that the $\mathscr{J}$-classes are linearly ordered. If $k S$ is left hereditary, then the intersection of any two principal left ideals of $S$ is either empty or itself principal.

If, in addition, the ring $k$ is noetherian (e.g., if $k$ is a field), then $k S$ is right hereditary also, so that the intersection of any two principal right ideals is either empty or again principal.

Proof. The last part of the theorem follows from the first part by observing that $k$ noetherian and $S$ finite imply that $k S$ is noetherian and that right and left global dimension are equal for noetherian rings (see, e.g., [3]).

To prove the first part we see first that since $S$ is a union of groups, principal left ideals have idempotent generators. Let $e_{1}, e_{2} \in S$ be idempotents. Then $k S e_{1}+k S e_{2}$ is $k S$-projective since $k S$ is left hereditary. Hence there exists an idempotent $\gamma \in k S e_{1} \cap k S e_{2}$ such that $k S e_{1} \cap k S e_{2}=k S \gamma$. 
Also, by Corollary 3 above, if $S e_{1} \cap S e_{2} \neq \varnothing$, the $\mathscr{L}$-classes $L$ of $S$ maximal with respect to $L \subset S e_{1} \cap S e_{2}$ lie in distinct $\mathscr{J}$-classes of $S$. Then the proof is concluded by the following lemma.

LEMmA. If $S$ is a union of groups monoid whose $\mathscr{J}$-classes are linearly ordered and if $I \subset S$ is a left ideal such that the $\mathscr{L}$-classes $L$ of $S$ maximal with respect to $L \subset I$ lie in distinct $\mathscr{J}$-classes of $S$, then $k I$ has an idempotent generator if and only if $I$ is principal.

ProOF. If $I$ is a principal left ideal of $S$ it has an idempotent generator in $S$, which will also serve as an idempotent generator for $k I$ in $k S$.

Now suppose we have $I$ and an idempotent $\gamma=\gamma^{2} \in k I$ such that $k I=$ $k S \gamma$. Suppose that $L_{1}$ and $L_{2}$ are distinct $\mathscr{L}$-classes of $S$ maximal with respect to $L_{i} \subset I$. Then $L_{1}$ and $L_{2}$ lie in distinct $\mathscr{J}$-classes of $S$, say $L_{i} \subset J_{i}$, $i=1,2$, and $J_{1} \geqq J_{2}$ in the $\mathscr{J}$-class ordering. Let $I^{\prime}=I \backslash\left(L_{1} \cup L_{2}\right)$. Then $I^{\prime}$ is a left ideal of $S$. We can write $\gamma=\gamma_{1}+\gamma_{2}+\gamma^{\prime}$ where $\gamma_{i}$ is a $k$-linear combination of elements of $L_{i}$ for $i=1,2$, and $\gamma^{\prime}$ is a $k$-linear combination of elements of $I^{\prime}$. Let $e_{i} \in L_{i}$ be idempotents for $i=1,2$. Now $e_{1} e_{2} \mathscr{J} e_{2} e_{1} \mathscr{J} e_{2}$ since $S$ is a union of groups (see [2, Theorem 4.4]). Hence we know that $e_{1} e_{2} \mathscr{L} e_{2}$ so that since $J_{1}$ and $J_{2}$ are completely simple, $e_{1} L_{2} \subset L_{2}$ and $e_{i} L_{i} \subset L_{i}$ for $i=1,2$. The maximality of $L_{2}$ and the fact that $e_{2} e_{1} \mathscr{J} e_{2}$ gives $e_{2} L_{1} \cap L_{2}=\varnothing$.

Now $e_{1}=e_{1} \gamma=e_{1} \gamma_{1}+e_{1} \gamma_{2}+e_{1} \gamma^{\prime}$ implies that $e_{1} \gamma_{1}=e_{1}, e_{1} \gamma_{2}=0$, and $e_{1} \gamma^{\prime}=0$ since elements of $S$ are linearly independent over $k$. Thus if $\gamma_{2}=$ $\sum_{y \in L_{2}} m_{y} y, m_{y} \in k$, this will imply that $\sum_{y \in L_{2}} m_{y}=0$. But also $e_{2}=e_{2} \gamma=$ $e_{2} \gamma_{1}+e_{2} \gamma_{2}+e_{2} \gamma^{\prime}$ implies that $e_{2} \gamma_{2}=e_{2}$, since $e_{2} \gamma_{2}$ is a $k$-linear combination of elements of $L_{2}$ while $e_{2} \gamma_{1}+e_{2} \gamma^{\prime}$ is a $k$-linear combination of elements of $I \backslash L_{2}$. However, $e_{2}=e_{2} \gamma_{2}$ implies that $\sum_{y \in L_{2}} m_{y}=1$, contradiction. Thus it is impossible that there be two distinct $\mathscr{L}$-classes of $S$ maximal with respect to containment in $I$. Thus there is a unique such $\mathscr{L}$-class, say $L_{1}$, which then says that $I=S e_{1}$.

\section{REFERENCES}

1. M. Arbib (Editor), Algebraic theory of machines, languages, and semigroups, Academic Press, New York, 1968. MR 38 \#1198.

2. A. H. Clifford and G. B. Preston, The algebraic theory of semigroups. Vol. 1, Math. Surveys, no. 7, Amer. Math. Soc., Providence, R.I., 1961. MR 24 \#A2627.

3. J. P. Jans, Rings and homology, Holt, Rinehart and Winston, New York, 1964. MR 29 \#1243.

4. W. R. Nico, Homological dimension in semigroup algebras, J. Algebra 18 (1971), 404-413. MR 43 \#3359.

5. —_, An improved upper bound for global dimension in semigroup algebras, Proc. Amer. Math. Soc. 35 (1972), 34-36.

Department of Mathematics, Tulane University, New Orleans, louisiana 70118 